\title{
A PRIORI ESTIMATES AND UNIQUE CONTINUATION THEOREMS FOR SECOND ORDER PARABOLIC EQUATIONS $\left({ }^{1}\right)$
}

\author{
BY \\ RAYMOND JOHNSON
}

\begin{abstract}
It is shown that solutions of second-order linear parabolic equations subject to global constraint satisfy an a priori estimate which has among its consequences that if a solution of such an equation vanishes on the characteristic $t=T$ and satisfies the global constraint, it vanishes identically.
\end{abstract}

1. Introduction. The continuation of solutions of a parabolic equation backwards in time is known to be an unstable problem. If global constraints (the type varying from problem to problem) are specified, the problem can be stabilized and a priori bounds derived. The paper of Douglas and Cannon [3] treated the problem of finding a numerical approximation to a function satisfying the heat equation given the values of the function on some subset of the half-space, where the values are known to be approximated within a given error. A priori estimates on the solution allowed the formation of numerial approximates to the solution.

In this paper, the basic a priori estimate is extended to solutions of more general second order equations. As in the paper of Douglas and Cannon [3], the bound can be applied to construct and bound a numerical approximation to a solution of the parabolic equation. The bound also implies a unique continuation theorem for solutions of the parabolic equations.

As in Friedman [4] the operator is a second-order uniformly parabolic operator with bounded, uniformly Hölder continuous coefficients; hence, the classical fundamental solution, denoted $\Gamma(x, t ; \xi, \tau)$, for $x, \xi \in \boldsymbol{R}_{n}, 0 \leqq \tau<t<+\infty$, exists. The additional restrictions on the coefficients will be given below in the statement of the a priori estimate which is the main result.

Received by the editors June 3, 1970 and, in revised form, November 1, 1970.

AMS 1969 subject classifications. Primary 3562; Secondary 3507.

Key words and phrases. Second-order parabolic equations, a priori estimates, unique continuation, backwards in time, global constraint, fundamental solution.

(1) This paper contains essentially the results of the author's thesis at Rice University under Professor Jim Douglas, Jr., while the author was supported by a N.S.F. Fellowship. 
THEOREM 1. Consider the differential operator

$$
L u=\sum_{i, j=1}^{n} a_{i j}\left(x_{j}\right) u_{x_{i} x_{j}}+\sum_{i=1}^{n} b_{i}(x, t) u_{x_{i}}+c(x, t) u-u_{t},
$$

where the coefficients are as above, and further,

(1) $a_{i j}$ do not depend on time;

(2) $b_{i}, c$ have extensions in the time variables as holomorphic functions of $\sigma$ in $\operatorname{Re} \sigma>0$, with the preservation of the boundedness and uniform Hölder continuity.

If $u$ is a solution of $L u=0$, which is of the form

$$
u=\int \Gamma(x, t ; \xi, 0) d \mu(\xi)
$$

where $\mu$ is a measure of slow increase, and if $|u(x, T)| \leqq \varepsilon$, for each $x \in R^{n}$, then for any compact set $K \subseteq R^{n} x(0, T)$, there are constants $C(K), 0<\alpha(K)<1$, such that

$$
\sup _{(x, t) \in K}|u(x, t)| \leqq C(K) \varepsilon^{\alpha} M^{1-\alpha},
$$

where $M$ arises from a seminorm associated with $\mu$.

This is the statement of the theorem in its full generality. The interesting case is that in which $b_{i}$ and $c$ are also independent of time. It is also applicable when $b_{i}(x, t)=b_{i}(x) f_{i}(t)$, where $f_{i}$ is analytic for $\operatorname{Re} \sigma>0$, and $c$ satisfies an analogous condition.

The proof of Theorem 1 will be given in $\S 3$, following necessary preliminaries in $\S 2$, which also contains a description of how these preliminaries fit into the proof. $\S 4$ contains remarks on and applications of the main theorem. In $\S 5$, the main technical tool is presented.

2. Preliminaries. A natural way of proving Theorem 1 would be to show that the function $t \rightarrow|u(x, t)|$ is $\log$ convex. As indicated by the hypotheses of analyticity, it will be shown that $u$ can be extended as a holomorphic function $u(x, \sigma)$ for $\operatorname{Re} \sigma>0$. Then use will be made of the fact that $\sigma \rightarrow|u(x, \sigma)|$ is $\log$ subharmonic.

Since

$$
u(x, t)=\int_{R_{n}} \Gamma(x, t ; \xi, 0) d \mu(\xi)
$$

the obvious way to extend $u$ is by

$$
u(x, \sigma)=\int_{\boldsymbol{R}_{\boldsymbol{n}}} \Gamma(x, \sigma ; \xi, 0) d \mu(\xi), \quad \operatorname{Re} \sigma>0 .
$$

For this to be valid, it must be shown that $\Gamma$ admits such an extension under the hypotheses on the coefficients of $L$ stated in Theorem 1 . The following theorem provides such an extension. 
THEOREM 2. For the differential operator

$$
L u=\sum_{i, j=1}^{n} a_{i j}(x) \frac{\partial^{2} u}{\partial x_{i} \partial x_{j}}+\sum_{i=1}^{n} b_{i}(x, t) \frac{\partial u}{\partial x_{i}}+c(x, t) u-\frac{\partial u}{\partial t},
$$

assume that the coefficients satisfy the hypotheses of Theorem 1. Then $\Gamma$, the fundamental solution of $L$, can be extended as a holomorphic function of $\sigma$ for $\operatorname{Re} \sigma>0$ with the bound

$$
|\Gamma(x, \sigma ; \xi, 0)| \leqq C(\delta) \exp \left(-\frac{(1-s)|x-\xi|^{2} \operatorname{Re} \sigma}{4 \bar{\lambda}_{1}|\sigma|^{2}}\right),
$$

for any $s, 0<s<1$, if $|\arg \sigma| \leqq(\pi / 2)-\delta$.

A precise estimate for $C$ can be given with the proof of this theorem in $\S 5$. If $a_{i j}(x)=\delta_{i j}$ and $b_{i}(x, t)=0$, the bound is sharper. Then

$$
|\Gamma(x, \sigma ; \xi, 0)| \leqq C(\delta) \exp \left(-|x-\xi|^{2} / 4|\sigma|\right) \quad \text { if }|\arg \sigma| \leqq(\pi / 2)-\delta .
$$

Now that it is known that $u$ can be extended as a holomorphic function of $\sigma$, the time variable, for $\operatorname{Re} \sigma>0$, it follows that for fixed $x$, the function $\sigma \rightarrow \ln |u(x, \sigma)|$ is subharmonic.

The next sequence of results guarantees that if $u$ can be bounded "crudely" on three sides of a rectangle in the complex plane and "sharply" on the fourth side, it can be bounded "sharply" on the perpendicular bisector of the fourth side. An outline of what happens at a fixed $x$ is the following.

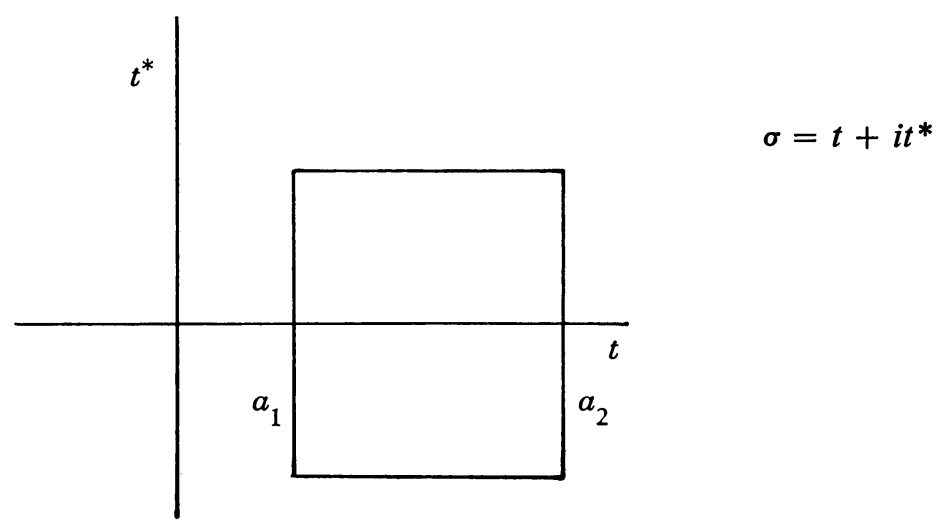

A sharp bound on $t^{*}=0$ for $t \geqq a_{1}$ will follow from the maximum principle. A crude bound in the remainder of the rectangle is a consequence of the properties of $\mu$ and the derived bound on $\Gamma$. A sharp bound on the perpendicular bisector of $a_{1} \leqq t \leqq a_{2}$, that is on $\left(a_{1}+a_{2}\right) / 2+i t^{*}$, follows because $|u|$ is log subharmonic. Considering the upper and lower rectangles separately, we now have a bound on $\left(a_{1}+a_{2}\right) / 2+i t^{*},\left|t^{*}\right| \leqq N$. 


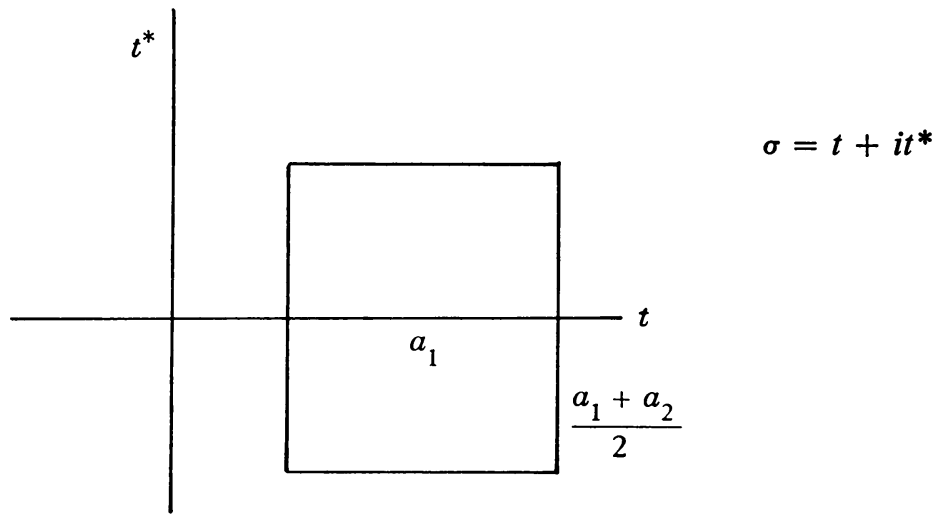

We now have a sharp bound on $\left(a_{1}+a_{2}\right) / 2+i t^{*}$ and, by hypothesis, a crude bound on the remainder of the rectangle. Applying the above mentioned property will give us a sharp bound on $t^{*}=0, \delta \leqq t \leqq\left(a_{1}+a_{2}\right) / 2$, where $\delta$ arbitrary. Thus the known bound for $t \geqq a_{1}$ is extended to a bound for $t \leqq a_{1}$.

The desired property of subharmonic functions is essentially contained in the paper of Douglas and Cannon [3]. It is stated in terms of negative bounds because it is to be applied to $\ln |u|$.

THEOREM 3. Suppose $v$ is subharmonic in an open subset $\Omega$ of $\boldsymbol{R}_{2}$ and that $K=$ $\left\{(x, y)\left|a_{1} \leqq y \leqq a_{2},\right| x \mid \leqq \beta\right\}$ is contained in $\Omega$. If $v\left(x, a_{1}\right) \leqq M \leqq 0$, and on the remainder of $\partial K, v \leqq 0$, it follows that

$$
v(0, y) \leqq M \sinh \left(\left[\pi\left(a_{2}-y\right)\right] / 2 \beta\right)\left(\sinh \left[\pi\left(a_{2}-a_{1}\right) / 2 \beta\right]\right)^{-1},
$$

for $a_{1} \leqq y \leqq a_{2}$.

Proof. $v\left(x, a_{1}\right) \leqq M \cos (\pi x / 2 \beta)$ because $M \leqq 0,|x| \leqq \beta$. On the remainder of $\partial K, v \leqq 0$. Therefore, on $\partial K, v \leqq h$, where $h$ is a harmonic function which equals $M \cos (\pi x / 2 \beta)$ on $y=a_{1}$ and 0 on the remainder of $\partial K$. Then, $v \leqq h$ in $K$. But $h$ is easily seen to be

$$
h(x, y)=[M \cos (\pi x / 2 \beta)] \sinh \left(\pi\left(a_{2}-y\right) / 2 \beta\right) / \sinh \left(\pi\left(a_{2}-a_{1}\right) / 2 \beta\right) .
$$

It follows immediately that if $K$ is taken in the form

$$
K=\left\{(x, y) \mid a_{1} \leqq y \leqq a_{2}, b_{1} \leqq x \leqq b_{2}\right\},
$$

and it is assumed that $v\left(x, a_{1}\right) \leqq M \leqq 0$, then

$$
v\left(\left(b_{1}+b_{2}\right) / 2, y\right) \leqq M \sinh \left(\pi\left(a_{2}-y\right) / b_{2}-b_{1}\right) / \sinh \left(\pi\left(a_{2}-a_{1}\right) / b_{2}-b_{1}\right) .
$$

It is also clear that if the bound is instead assumed on $x=b_{1}$, the conclusion becomes

$$
u\left(x,\left(a_{1}+a_{2}\right) / 2\right) \leqq M \sinh \left(\pi\left(b_{2}-x\right) / a_{2}-a_{1}\right) / \sinh \left(\pi\left(b_{2}-b_{1}\right) / a_{2}-a_{1}\right) .
$$

In order to get the process described above started, a bound for $u$ on a segment of the real axis is needed. If it is assumed that $|u(x, T)| \leqq \varepsilon$ for all $x \in \boldsymbol{R}_{n}$, as in 
Theorem 1, this bound follows from the maximum principle for unbounded regions as given in Il'in, Kalašnikov, and Oleĭnik [7, p. 13].

THEOREM 3. Consider

$$
L u=\sum_{i, j=1}^{n} a_{i j}(x, t) \frac{\partial^{2} u}{\partial x_{i} \partial x_{j}}+\sum_{i=1}^{n} b_{i}(x, t) \frac{\partial u}{\partial x_{j}}+c(x, t) u-\frac{\partial u}{\partial t},
$$

where $\left[a_{i j}\right]$ is positive semidefinite, $\left|a_{i j}\right| \leqq M,\left|b_{i}\right| \leqq M(1+|x|),|c| \leqq M(1+|x|)^{2}$, and $c \leqq 0$. Suppose that $u$ is continuous on $\boldsymbol{R}_{n} \times[0, T]$ and twice continuously differentiable in $\boldsymbol{R}_{n} \times(0, T]$, and that $L u \leqq 0$ and $u(x, t) \geqq-B \exp \left(\beta|x|^{2}\right)$. If $u(x, 0) \geqq 0$, then $u(x, t) \geqq 0$ for $0 \leqq t \leqq T$.

In our case the assumption is that $|u(x, T)| \leqq \varepsilon$; to apply the above theorem we translate in $t$ and apply it to $\pm u+\varepsilon$.

Corollary. Let $L$ be as above and $u$ be continuous in $R_{n} \times\left[T, T_{0}\right]$ and twice continuously differentiable in $\boldsymbol{R}_{n} \times\left(T, T_{0}\right]$ such that $L u=0$ and

$$
|u(x, t)| \leqq B \exp \left(\beta|x|^{2}\right), \quad T \leqq t \leqq T_{0} .
$$

If $|u(x, T)| \leqq \varepsilon$, then $|u(x, t)| \leqq \varepsilon$ for $T \leqq t \leqq T_{0}$.

Proof. Let $v^{ \pm}(x, t)= \pm u(x, t+T)+\varepsilon$. For $0 \leqq t \leqq T_{0}-T$,

$$
L\left(v^{ \pm}\right)=L( \pm u)+L \varepsilon=c \varepsilon \leqq 0,
$$

thus satisfying one of the conditions of Theorem 3 . The hypotheses required of the coefficients have been assumed. It also follows that

$$
v^{ \pm}(x, t) \geqq-\varepsilon-B \exp \left(\beta|x|^{2}\right) \geqq-(B+\varepsilon) \exp \left(\beta|x|^{2}\right) .
$$

Finally, $v^{ \pm}(x, 0)= \pm u(x, T)+\varepsilon \geqq 0$. Therefore, $v^{ \pm}(x, t) \geqq 0,0 \leqq t \leqq T_{0}-T$; that is, $|u(x, t)| \leqq \varepsilon, T \leqq t \leqq T_{0}$.

The requirement that $c \leqq 0$ can be removed by the well-known device of replacing $u$ by

$$
v(x, t)=u(x, t) \exp (-L t)
$$

where $L$ is an upper bound for $c$.

Suppose that $u$ is as in Theorem 1 ; that is $L u=0$ and

$$
u(x, t)=\int_{R_{n}} \Gamma(x, t ; \xi, 0) d \mu(\xi),
$$

where $\mu$ is a measure of slow increase. The assumptions on the coefficients clearly imply those needed in Theorem 2 and Theorem 3. To verify that Theorem 3 is applicable, it must be shown that given $T \leqq T_{0}$, there is a $B, \beta>0$ such that

But

$$
|u(x, t)| \leqq B \exp \left(\beta|x|^{2}\right) .
$$

$$
|\Gamma(x, t ; \xi, 0)| \leqq C t^{-n / 2} \exp \left(-\lambda_{0}^{*}|x-\xi|^{2} / 4 t\right),
$$


where $\lambda_{0}^{*}$ is any positive real number $<1 / \bar{\lambda}_{0}$, and since $\mu$ is of slow increase, there is a positive $l$ such that

$$
\int_{R_{n}}\left(1+|\xi|^{2}\right)^{-l} d|\mu|(\xi)<+\infty
$$

Taking the smallest such $l$, it follows that

$$
\begin{aligned}
|u(x, t)| & \leqq \int_{R_{n}}|\Gamma(x, t ; \xi, 0)|\left(1+|\xi|^{2}\right)^{l}\left(1+|\xi|^{2}\right)^{-l} d|\mu|(\xi), \\
& \leqq M \sup _{R_{n}}\left(1+|\xi|^{2}\right)^{+l}|\Gamma(x, t ; \xi, 0)|,
\end{aligned}
$$

and the bound on $\Gamma$ and Cauchy's inequality imply

$$
\begin{aligned}
|\Gamma(x, t ; \xi, 0)| & \leqq C T^{-n / 2} \exp \left(-\lambda_{0}^{*}|x-\xi|^{2} / 4 T_{0}\right), \\
& \leqq C T^{-n / 2} \exp \left(\lambda_{0}^{*}|x|^{2} / 2 T_{0}\right) \exp \left(-\lambda_{0}^{*}|\xi|^{2} / 8 T_{0}\right), \quad T \leqq t \leqq T_{0},
\end{aligned}
$$

which implies that

$$
\left(1+|\xi|^{2}\right)^{l}|\Gamma(x, t ; \xi, 0)| \leqq C T^{-n / 2}\left(8 T l / e \lambda_{0}^{*}\right)^{l} \exp \left(\lambda_{0}^{*} / 4 T_{0}\right) \exp \left(\lambda_{0}^{*}|x|^{2} / 2 T_{0}\right),
$$

where $C$ can be made precise if necessary. Hence,

$$
|u(x, t)| \leqq C M \exp \left(\lambda_{0}^{*}|x|^{2} / 2 T_{0}\right) \quad \text { if } T \leqq t \leqq T_{0},
$$

where $C$ can be made precise if necessary.

3. Proof of the main theorem. The proof of the main theorem now follows easily. For the statement of this theorem, see $\S 1$.

It suffices to take $K$ in the form $\left\{(x, t)|\kappa \leqq t \leqq T| x \mid, \leqq X_{1}\right\}$, where $X_{1}$ and $\kappa$ are arbitrary positive real numbers.

By Theorem 1, we can extend $\Gamma$ to $\operatorname{Re} \sigma>0$, and thus can define

$$
u(x, \sigma)=\int_{R_{n}} \Gamma(x, \sigma ; \xi, 0) d \mu(\xi) .
$$

It follows, as at the end of Theorem 3 , that $|u(x, \sigma)| \leqq C M \exp \left(\beta|x|^{2}\right)$, $T_{1} \geqq \operatorname{Re} \sigma \geqq \frac{1}{2} \kappa,|\operatorname{Im} \sigma| \leqq T^{*}$. Therefore,

$$
|u(x, \sigma)| \leqq C M \quad \text { for } T_{1} \geqq \operatorname{Re} \sigma \geqq \frac{1}{2} \kappa,|\operatorname{Im} \sigma| \leqq T^{*},|x| \leqq X_{1} .
$$

Fix $x$ with $|x| \leqq X_{1}$, and let $T_{1}$ be arbitrary finite with $T_{1}>T$. Let $Q_{1}$ $=\left\{z \mid T \leqq \operatorname{Re} z \leqq T_{1}, 0 \leqq \operatorname{Im} z \leqq T^{*}\right\}$. The function $\sigma \rightarrow \ln (|u(x, \sigma)| / C M)$ is subharmonic on $Q_{1}$, and $\ln (|u(x, \sigma)| / C M) \leqq 0$ on $Q_{1}$.

By Theorem 3,

$$
\ln (|u(x, z)| / C M) \leqq \ln \left(\varepsilon \exp \left(M_{0}\left(T_{1}-T\right)\right) / C M\right) \text { for } \operatorname{Im} z=0,
$$

where $M_{0}$ is an upper bound of $C$ for $T \leqq t \leqq T_{1}$. It can be assumed that

$$
\ln \left(\varepsilon \exp \left(M_{0}\left(T_{1}-T\right)\right) / C M\right) \leqq 0,
$$


because otherwise, $C M \leqq \varepsilon \exp \left(M_{0}\left(T_{1}-T\right)\right)$, and the desired bound for $u$ follows.

By the second remark after Theorem 2,

$$
\ln \left(\left|u\left(x,\left(T_{1}+T\right) / 2+i t^{*}\right)\right| / C M\right) \leqq \ln \left(\varepsilon \exp \left(M_{0}\left(T_{1}-T\right)\right) / C M\right) \alpha\left(t^{*}\right),
$$

where $\alpha\left(t^{*}\right)=\sinh \left(\pi\left(T_{1}^{*}-t^{*}\right) /\left(T_{1}-T\right)\right) / \sinh \left(\pi T^{*} /\left(T_{1}-T\right)\right)$.

The same argument can be applied to $Q_{2}=\left\{z \mid T \leqq \operatorname{Re} z \leqq T_{1},-T^{*} \leqq \operatorname{Im} z \leqq 0\right\}$, and the same result holds with $\alpha$ replaced by $\gamma$, where

$$
\gamma\left(t^{*}\right)=\sinh \left(\pi\left(t^{*}+T^{*}\right) /\left(T_{1}-T\right)\right) / \sinh \left(\pi T^{*} /\left(T_{1}-T\right)\right) .
$$

Therefore,

$$
\ln \left(\left|u\left(x,\left(T+T_{1}\right) / 2+i t^{*}\right)\right| / C M\right) \leqq \ln \left(\varepsilon \exp \left(M_{0}\left(T_{1}-T\right)\right) / C M\right) \rho\left(t^{*}\right),
$$

where

$$
\begin{aligned}
\rho\left(t^{*}\right) & =\alpha\left(t^{*}\right), & & t^{*} \geqq 0, \\
& =\gamma\left(t^{*}\right), & & t^{*} \leqq 0 .
\end{aligned}
$$

Let $Q^{\prime}=\left\{z\left|\frac{1}{2} \kappa \leqq \operatorname{Re} z \leqq\left(T+T_{1}\right) / 2,\right| \operatorname{Im} z \mid \leqq T_{2}^{*}\right\}$, where $T_{2}^{*}<T_{1}^{*}$. Since

$$
\begin{aligned}
\ln \left(\left|u\left(x,\left(T+T_{1}\right) / 2+i t^{*}\right)\right| / C M\right) & \leqq \ln \left(\varepsilon \exp \left(M_{0}\left(T_{1}-T\right)\right) / C M\right) \rho\left(t^{*}\right) \\
& \leqq \ln \left(\varepsilon \exp \left(M_{0}\left(T_{1}-T\right)\right) / C M\right) \rho\left(T_{2}^{*}\right),
\end{aligned}
$$

for $\sigma \in \partial Q^{\prime} \cap\left\{z \mid \operatorname{Re} z=\frac{1}{2}\left(T+T_{1}\right)\right\}$, and it is known already that $\ln (|u(x, \sigma)| / C M) \leqq$ 0 for $\sigma \in \partial Q^{\prime}$, the remark after Theorem 2 implies

$$
\ln (|u(x, t)| / C M) \leqq \ln \left(\varepsilon \exp \left(M_{0}\left(T_{1}-T\right)\right) / C M\right) \rho\left(T_{2}^{*}\right) \beta(t),
$$

for $\frac{1}{2} \kappa \leqq t \leqq \frac{1}{2}\left(T+T_{1}\right)$, where

$$
\beta(t)=\sinh \left(\frac{\pi\left(\left(T+T_{1}\right) / 2-t\right)}{2 T_{2}^{*}}\right) / \sinh \frac{\pi\left(T+T_{1}-\kappa\right)}{4 T_{2}^{*}},
$$

and hence

$$
\ln (|u(x, t)| / C M) \leqq \ln \left(\varepsilon \exp \left(M_{0}\left(T_{1}-T\right)\right) / C M\right) \rho\left(T_{2}^{*}\right) \beta(\kappa),
$$

for $\kappa \leqq t \leqq T$. This is equivalent to

$$
|u(x, t)| \leqq(C M)^{1-\rho\left(T_{2}^{*}\right) \beta(x)}\left[\varepsilon \exp \left(M_{0}\left(T_{1}-T\right)\right)\right]^{\rho\left(T_{2}^{*}\right) \beta(x)},
$$

and finally

$$
|u(x, t)| \leqq C M^{1-\beta} \varepsilon^{\beta},
$$

where $\beta=\rho\left(T_{2}^{*}\right) \beta(\kappa)$, and thus $0<\beta<1$.

If more is known about the coefficients, it is possible to maximize $\beta$ by varying $T_{1}$ and $\kappa$.

4. Applications. The application of this bound to problems of numerical approximation proceeds exactly as in the paper of Douglas and Cannon [3]. The 
approximation is constructed as a linear combination of the values of the fundamental solution with the coefficients of the expansion being determined by linear programming. The difficulty with the method will be in determining an accurate numerical approximation to the fundamental solution.

The following unique continuation theorem is an immediate corollary of the estimate.

THEOREM 4. Let

$$
L u=\sum_{i, j=1}^{n} a_{i j}(x) \frac{\partial^{2} u}{\partial x_{i} \partial x_{j}}+\sum_{i=1}^{n} b_{i}(x) \frac{\partial u}{\partial x_{i}}+c(x) u-\frac{\partial u}{\partial t},
$$

where $a_{i j}, b_{i}, c$ are defined on $\boldsymbol{R}_{n}$. Suppose that $L$ is uniformly parabolic; that is, there exist $\bar{\lambda}_{0} \geqq 0, \bar{\lambda}_{1}$ such that

$$
\bar{\lambda}_{0}|\xi|^{2} \leqq \sum_{i, j=1}^{n} a_{i j}(x) \xi_{i} \xi_{j} \leqq \bar{\lambda}_{1}|\xi|^{2} \quad \text { for all } \xi \in \boldsymbol{R}_{n}, x \in \boldsymbol{R}_{n}
$$

The matrix $a_{i j}$ is assumed symmetric. Assume that $a_{i j}, b_{i}$, and $c$ are uniformly Hölder continuous with exponent $\alpha$, and are uniformly bounded. Suppose that $L u=0$, where $u$ is given by

$$
u(x, t)=\int_{R_{n}} \Gamma(x, t ; \xi, 0) d \mu(\xi) .
$$

Then if $u(x, T)=0$ for $x \in \boldsymbol{R}_{n}$, $u$ is identically zero.

Here $\Gamma$ is the fundamental solution of $L$, while $\mu$ is a measure of slow increase. Such theorems have been proven by Yosida [14], Mizohata [12], and Ito and Yamabe [8].

Thus, if one knows conditions which guarantee the representation (1), unique continuation follows. Here we list a few such conditions. By a solution of the equation, we understand a classical solution in $\boldsymbol{R}_{n} \times(0, \infty)$.

COROLlaRY 1. If the coefficients satisfy the above hypotheses and if $u$ is a solution of $L u=0$ in $\boldsymbol{R}_{n} \times(0, \infty)$, which is continuous in $\boldsymbol{R}_{n} \times[0, \infty)$, with $u(x, 0)=\varphi(x)$, where $\varphi$ is a bounded continuous function on $\boldsymbol{R}_{n}$, then

$$
u(x, t)=\int_{R_{n}} \Gamma(x, t ; \xi, 0) \varphi(\xi) d \xi .
$$

Proof. This is a consequence of Theorem 12, p. 25, and Theorem 10, p. 44 of Friedman [4].

COROLlaRY 2. If $u$ is a solution of the heat equation in $\boldsymbol{R}_{n} \times(0, \infty)$ and if $\int_{R_{n}}|u(x, t)|^{p} d x \leqq C^{p}$ for $0<t<+\infty$, with some $p, 1<p<+\infty$, then

$$
u(x, t)=\int k(x-\xi, t) f(\xi) d \xi, \text { for some } f \in L^{p}\left(\boldsymbol{R}_{n}\right),
$$


where $\|f\|_{p} \leqq C$. If $p=1$, then

$$
u(x, t)=\int k(x-\xi, t) d \mu(\xi)
$$

where $\mu$ is an integrable measure and $\int|d \mu| \leqq C$.

Proof. See Jones [9], where a slightly more general result is proven.

This result can be extended to the general second order linear equation. See Johnson [15].

Such solutions have the property of unique continuation backwards in time because

$$
\int \frac{|f(\xi)|}{\left(1+|\xi|^{2}\right)^{l}} d \xi \leqq\left(\int|f(\xi)|^{p} d \xi\right)^{1 / p}\left(\int\left(1+|\xi|^{2}\right)^{-l p^{\prime}} d \xi\right)^{1 / p^{\prime}}
$$

and any choice of $l p^{\prime}>n / 2$ insures that the measure defined by $f$ is of slow increase.

One can also apply the theorem to positive solutions of parabolic equations, using results of Widder [13], Krzyżański [10] and Guenther [6], if conditions are imposed to guarantee that the boundary measure is a tempered distribution.

There are also results known for operator equations, [1], [2], but because of the difficulty of comparison of assumptions, these results can be compared with theorems for classical solutions, due to Landis [11] and Glagoleva [5]. All of these results require at least $C^{1,1}$ coefficients in the principal part, which is improved here to $C^{0, \alpha}$, when these coefficients are independent of time. The form of the global constraint is very flexible.

5. Technical lemma. This section contains the proof of Theorem 2, which is stated in $\$ 2$. The hypotheses on the coefficients are those stated in $\$ 2$. The proof is a straightforward adaptation of the proof of the existence of a fundamental solution, as presented in Friedman [4, pp. 1-18]. For $x \in \boldsymbol{R}_{n}, \xi \in \boldsymbol{R}_{n}$ and $t-\tau>0$, it is known that, if $\Gamma$ denotes the fundamental solution,

$$
\Gamma(x, t ; \xi, \tau)=Z(x, t ; \xi, \tau)+\int_{\tau}^{t} \int_{R_{n}} Z(x, t ; \eta, \rho) \Phi(\eta, \rho ; \xi, 0) d \eta d \rho,
$$

where $Z$ is a parametrix and $\Phi$ is given by

$$
\Phi(x, t ; \xi, \tau)=\sum_{v=1}^{\infty}(L Z)_{v}(x, t ; \xi, \tau)
$$

where

$$
(L Z)_{1}(x, t ; \xi, \tau)=L_{x, t} Z(x, t ; \xi, \tau)
$$

and

$$
(L Z)_{v+1}(x, t ; \xi, \tau)=\int_{\tau}^{t} \int_{R_{n}} L Z(x, t ; \eta, \rho)(L Z)_{v}(\eta, \rho ; \xi, \tau) d \eta d \rho
$$


If $\Gamma$ is to be extended in the time variable to $\sigma$ complex for $\operatorname{Re} \sigma>0$, the form of the expansion is obvious by the above. It should be the case that

$$
\Gamma(x, \sigma ; \xi, \gamma)=Z(x, \sigma ; \xi, \gamma)+\int_{\gamma}^{\sigma} \int_{R_{n}} Z(x, \sigma ; \eta, \rho) \Phi(\eta, \rho ; \xi, \gamma) d \eta d \rho,
$$

for $x \in \boldsymbol{R}_{n}, \xi \in \boldsymbol{R}_{n}$ and $\operatorname{Re}(\sigma-\gamma)>0$, where $\Phi$ is given by

with

$$
\Phi(x, \sigma ; \xi, \gamma)=\sum_{v=1}^{\infty}(L Z)_{v}(x, \sigma ; \xi, \gamma)
$$

$$
(L Z)_{1}(x, \sigma ; \xi, \gamma)=L_{x, \sigma} Z(x, \sigma ; \xi, \gamma),
$$

and

$$
(L Z)_{v+1}(x, \sigma ; \xi, \gamma)=\int_{\gamma}^{\sigma} \int_{R_{n}} L Z(x, \sigma ; \eta, \rho)(L Z)_{v}(\eta, \rho ; \xi, \gamma) d \eta d \rho .
$$

The proof divides naturally into three parts. It is shown that the series representing $\Phi$ converges absolutely and uniformly on compact subsets, and by deriving bounds on $\Phi$, it is shown that $\Gamma$ exists and can be bounded as in the real case. The remaining part, to prove that $\Gamma$ is holomorphic, splits into two parts. As $\Gamma$ is the sum of a parametrix, which can easily be seen to be holomorphic, and an extended volume potential of $\Phi$, it is shown that the volume potential of a function holomorphic in the time variable and Hölder continuous in the space variables is holomorphic in the time variable. The last step is the proof that $\Phi$ has these properties.

A sketch of the proofs will be given. The complete proofs can be found in the author's technical report at the University of Maryland [16]. Since the domain under consideration is unbounded, references to Friedman must be understood as adapted by $\S 6$, p. 22 . The convergence of the series defining $\Gamma$ for complex arguments follows from the adaptation of the bounds on the parametrix $Z$, given in Friedman, p. 16, (4.9-4.13), to the case in which the time variables depend on complex arguments. The complex variables must be restricted to carry this out; it must be assumed throughout that $|\arg (\sigma-\rho)| \leqq(\pi / 2)-\delta$, where $\delta>0$ and the bounds will depend on $\delta$.

Following Friedman, the volume potential of $f$, with respect to the parametrix $A$, is defined by

$$
V(x, \sigma)=\int_{\gamma}^{\sigma} \int_{R^{n}} Z(x, \sigma ; \eta, \rho) f(\eta, \rho) d \eta d \rho,
$$

where $\gamma$ is a complex number such that $\operatorname{Re} \gamma>0$ and $V$ is defined for all $\sigma$, with $\operatorname{Re}(\sigma-\gamma)>0$. It is shown that this function has the same differentiability properties as when $\sigma, \rho$ are real. Among the changes are the fact that when considering

$$
J(x, \sigma, \rho)=\int_{R^{n}} Z(x, \sigma ; \eta, \rho) f(\eta, \rho) d \eta
$$


the convergence of $J(x, \sigma, \rho)$ to $f(x, \sigma)$ is not proven for arbitrary $\rho$ converging to $\sigma$; only those for which $|\arg (\sigma-\rho)| \leqq(\pi / 2)-\delta$. Equivalents of Theorem 2 and 3 are proven under reasonable hypotheses on $f$; the equivalent of Theorem 4, p. 9 , again requires that $f$ be Hölder continuous in the space variables, as does the equivalent of Theorem 5, p. 12. The analogue of Theorem 7, p. 17, is then proven in exactly the same manner as in Friedman [4], and all the tools are now available to prove the analogue of Theorem 8, p. 19, which shows that the fundamental solution is holomorphic in $\sigma$ for $\operatorname{Re} \sigma>0$, and this concludes the proof.

\section{BIBLIOGRAPHY}

1. S. Agmon and L. Nirenberg, Properties of solutions of ordinary differential equations in Banach space, Comm. Pure Appl. Math. 16 (1963), 121-239. MR 27 \#5142.

2. - Lower bounds and uniqueness theorems for solutions of differential equations in a Hilbert space, Comm. Pure Appl. Math. 20 (1967), 207-229. MR 34 \#4665.

3. J. Cannon and J. Douglas, The approximation of harmonic and parabolic functions on half-spaces from interior data, Numerical Analysis of Partial Differential Equations, (C.I.M.E. $Z^{\circ}$ Ciclo, Ispra 1967), Edizioni Cremonese, Rome, 1968, pp. 193-230. MR 39 \#5076.

4. A. Friedman, Partial differential equations of parabolic type, Prentice-Hall, Englewood Cliffs, N. J., 1964. MR 31 \#6062.

5. R. Glagoleva, Some properties of the solutions of a linear second order parabolic equation, Mat. Sb. 74 (116) (1968), 47-74= Math. USSR Sb. 3 (1967), 41-67. MR 35 \#7003.

6. R. Guenther, Representation theorems for linear second-order parabolic partial differential equations, J. Math. Anal. Appl. 17 (1967), 488-501. MR 34 \#7977.

7. A. M. Il'in, A. S. Kalašnikov and O. A. Oleǐnik, Second order linear equations of parabolic type, Uspehi Mat. Nauk 17 (1962), no. 3 (105), 3-146=Russian Math. Surveys 17 (1962), no. 3, 1-143. MR 25 \#2328.

8. T. Ito and H. Yamabe, A unique continuation theorem for solutions of a parabolic differential equation, J. Math. Soc. Japan 10 (1958), 314-321. MR 20 \#5958.

9. B. F. Jones, Lipschitz spaces and the heat equation, J. Math. Mech. 18 (1968), 379-409.

10. M. Krzyżański, Sur les solutions non négatives de l'équation linéare normale parabolique, Rev. Roumaine Math. Pures Appl. 9 (1964), 393-408. MR 32 \#295.

11. E. M. Landis, Some questions in the qualitative theory of second-order elliptic equations (case of several independent variables), Uspehi Mat. Nauk 18 (1963), no. 1 (109), 3-62= Russian Math. Surveys 18 (1963), no. 1, 1-62. MR 27 \#435.

12. S. Mizohata, Le problème de Cauchy pour le passé pour quelques équations paraboliques, Proc. Japan Acad. 34 (1958), 693-696. MR 21 \#4293.

13. D. V. Widder, Positive temperatures on the infinite rod, Trans. Amer. Math. Soc. 55 (1944), 85-95. MR 5, 203.

14. K. Yosida, An abstract analyticity in time for solutions of a diffusion equation, Proc. Japan Acad. 35 (1959), 109-113. MR 21 \#4298.

15. R. Johnson, Representation theorems and Fatou theorems for second-order linear parabolic partial differential equations, Proc. London Math. Soc. (to appear).

16. - A priori estimates and unique continuation theorems for second-order parabolic equations, TR 70-160, University of Maryland, College Park, Md., 1970.

UNIVERSITY OF MARYLAND, College Park, Maryland 20742 